\title{
Correlación entre potencia y resistencia a la velocidad en una selección juvenil de fútbol
}

\section{Correlation between power and resistance to speed in a youth soccer team}

\author{
David Ricardo Medina-Samper¹ iD ; Milton Javier Pirazán-Rodríguez² (iD)
}

\begin{abstract}
1Universidad Pedagógica y Tecnológica de Colombia, Maestría en Pedagogía de la Cultura Física. Tunja - Boyacá, Colombia; davogol9@gmail.com

2Universidad Santo Tomás. Tunja - Boyacá, Colombia; miljapiro@hotmail.com
\end{abstract}

Cómo citar: Medina-Samper, D.R.; Pirazán-Rodríguez, M.J. 2022. Correlación entre potencia y resistencia a la velocidad en una selección juvenil de fútbol. Rev. Digit. Act. Fis. Deport. 8(1):e2178. http://doi.org/10.31910/rdafd.v8.n1.2022.2178

Artículo de acceso abierto publicado por Revista Digital: Actividad Física y Deporte, bajo una licencia Creative Commons CC BY-NC 4.0 Publicación oficial de la Universidad de Ciencias Aplicadas y Ambientales U.D.C.A, Institución de Educación Superior Acreditada de Alta Calidad por el Ministerio de Educación Nacional.

Recibido: agosto 30 de 2021 Aceptado: septiembre 28 de 2021 Editado por: Álvaro José Gracia Díaz

\section{RESUMEN}

Introducción: es preciso mencionar que el fútbol actual, del entorno nacional y regional, cuenta con algunas características físicas exigentes, lo que conlleva a que el deportista tenga unas condiciones adecuadas y pertinentes, para lograr afrontar las acciones que se dan en el juego. Objetivo: establecer la correlación que existe entre la potencia y resistencia a la velocidad en los jugadores de fútbol de categoría sub-17, del equipo profesional Patriotas Boyacá. Metodología: con enfoque empírico analítico y un diseño descriptivo correlacional no experimental, se les aplicó el test de Bosco, saltos Counter Mouvement Jump (CMJ), Repeat, Squat Jump (SJ), Ability Abalakov (ABK) y el test Sprint (RSA), con el fin de analizar las variables de estudio. Para el análisis estadístico, se adelantó un análisis exploratorio de datos, mediante estadística descriptiva; el procesamiento de la información, se realizó mediante en Excel y el software estadístico SPSS 13, analizando el coeficiente de correlación de Pearson ( $r$ ) datos paramétricos y un Alpha mayor o igual a 0,05. Resultados: en el test de Bosco, en (SJ), se encontró un promedio de $37,21 \mathrm{~cm}$; en $A B K$, se presenta una media de $43,27 \mathrm{~cm}$ y para
CMJ, se obtuvo una media de $37,97 \mathrm{~cm}$. Por otro lado, los resultados para el test RSA indican que el índice de fatiga de los deportistas obtuvo un promedio de 7,16 $\pm 4,50 \%$; este porcentaje indica que los futbolistas resultaron altamente heterogéneos en cuanto al IF, lo cual, refleja que no todos los deportistas están en excelentes valores, de acuerdo con la clasificación general del test. Conclusión: se aceptó la hipótesis nula, considerando, de esta manera, que no hubo correlación entre la potencia y resistencia a la velocidad.

Palabras claves: Potencia; Resistencia a la velocidad; Fútbol; Test de Bosco; Test RSA.

\section{ABSTRACT}

Introduction: It should be mentioned that current football in the national and regional environment has some physical and demanding characteristics, which lead to the athlete having optimal conditions to be able to face the actions that occur in the game. Objective: To stablish the correlation that exists between power and resistance to speed in soccer players sub-17 category in professional Boyacá's 
patriots' team. Methodology: Using an empiric analytic approach and a correlational descriptive design no experimental, the Bosco test was applied in the Counter Movement jump (CMJ), Repeat, Squat Jump (SJ), Ability Abalakov (ABK), and the Sprint test (RSA), with the aim of analyzing the study variables. For the statistic analysis there is a data exploratory analysis through descriptive statistic; The information data gathering was made by using Microsoft Excel and a statistic software SPSS 13, making the Pearson correlation coefficient ( $r$ ) parametric data and an over Alpha or equal to 0.05. Results: From the obtaining of Bosco test, in (SJ) it was found an average of 37.21 $\mathrm{cm}$, in $A B K$ it is a measure presented of $43,27 \mathrm{~cm}$ and for $\mathrm{CMJ}$ it was obtained a measure of $37.97 \mathrm{~cm}$. Of the test RSA, indicates that the fatigue index of the sport youngsters obtained an average of $7.16 \pm 4.50 \%$, this percentage indicates that soccer players result highly heterogeneous on terms of IF, which reflects that not every sport man is in excellent values according to the general classification on the test. Conclusion: The null hypothesis was accepted, considering on this way that there was not any correlation between power and resistance to speed.

Keywords: Power; Resistance to speed; Football (Soccer); Bosco test; RSA test.

\section{INTRODUCCIÓN}

Terrados et al. (2011) mencionan que "los deportes de equipo son una de las prácticas deportivas más importantes a nivel mundial" y algunos ejemplos de estos son el baloncesto, el balonmano, el fútbol, siendo este último el que mayor número de practicante tiene en el mundo, sobre todo a raíz de la creación de la FIFA, la cual, se encarga regular este deporte, desde 1904. Así, las cosas, desde una comprensión del concepto anteriormente mencionado, es importante resaltar, que la práctica deportiva es un fenómeno social que, a lo largo de los años, se ha manifestado en la comunidad, a través de escuelas de formación o equipos profesionales, brindado un espacio propicio para la consecución de beneficios personales o grupales.

Es pertinente mencionar, que el fútbol actual, del entorno nacional y regional, cuenta con algunas características físicas y exigencias, las cuales, conllevan a que el deportista tenga unas condiciones adecuadas y pertinentes, para lograr afrontar las acciones que se dan en el juego. Por tal motivo, es indispensable que haya una planificación de las necesidades actuales e individuales de un futbolista y, un ejemplo de lo anterior, es lo que mencionan Le Gall et al. (2002), quienes midieron las variables que interviene en la práctica deportiva y, de lo cual, explican que "la fuerza explosiva y la velocidad, que es dependiente de la fuerza, son factores determinantes en el rendimiento deportivo en los futbolistas", por lo tanto, la evaluación de estos parámetros es fundamental para determinar la situación actual de los deportistas y poder plantear su mejora y desarrollo.

También, es importante resaltar, que la rapidez con la que los futbolistas realizan las acciones propias del deporte, se relaciona con la fuerza explosiva y la velocidad, por ello, Salinero et al. (2013) dicen que "la velocidad solo es una capacidad derivada de la fuerza, mientras que la fuerza es una cualidad pura que determina la velocidad que adquieren los cuerpos al desplazarse, además, la fuerza explosiva en el fútbol habitualmente se ha relacionado con la ejecución de estos gestos técnicos y físicos tales como los saltos, los pases o los golpeos. La velocidad, no solo es fundamental su desarrollo por sí misma, sino también, es importante aplicarla en acciones con balón".

Por otra parte, hay que resaltar lo que mencionan Álvarez Medina et al. (2002), quienes explican que el fútbol se clasifica como un deporte de colaboraciónoposición, donde los miembros de un mismo equipo trabajan para conseguir un objetivo (colaboración) $y$, al mismo tiempo, se relacionan con los miembros del equipo contrario (oposición); sus acciones, se desarrollan en un espacio y tiempo veloz, cambiante y dinámico.

Del mismo modo, es importante resaltar lo que indica López (2017), en cuanto a que los esfuerzos que se realizan son acciones individuales, tales como el hacer remates, disputar balones cuerpo a cuerpo y soportar choques, al tiempo que se combinan con desplazamientos en línea recta o con cambio de dirección.

Por tanto, lo anterior se da en respuesta a situaciones de ataque o defensa, lo cual, exigen que el deportista tenga la capacidad para generar fuerza y recuperarse en el menor tiempo posible, siendo un equivalente de potencia o fuerza explosiva. 
Teniendo en cuenta lo anterior, es pertinente decir, que el resultado obtenido busca evidenciarse como estrategia, con el fin de describir y analizar la potencia de la resistencia de la velocidad de jugadores del equipo sub-17, de Patriotas Boyacá F.C. y, con ello, presentar los resultados de la investigación, para contribuir al mantenimiento de las variables de panificación de entrenamiento de forma individual y, a su vez, realizar aportes significativos al fútbol.

Es oportuno dejar claro que, si existiera alguna relación entre las dos variables, potencia y resistencia a la velocidad, ayudaría como aporte para los entrenamientos de los futbolistas, para que contribuya e intervenga en el desarrollo físico, en el desempeño en el terreno de juego y en las estrategias que mejoren el rendimiento. Igualmente, esta investigación permitirá complementar los resultados y conclusiones de la presente investigación.

\section{MATERIALES Y MÉTODOS}

Para el desarrollo de este estudio participaron 18 jugadores, con edad comprendida entre los 14,64 y 17,53 años, un peso mínimo de 58 kg y un máximo de $84 \mathrm{~kg}$, estatura mínima de 1,60 m y una estatura máxima de 1,85 m y un IMC de 22,61 $\pm 1,33 \mathrm{~kg}$, pertenecientes al equipo base de la categoría sub-17, quienes firmaron el consentimiento informado, antes de realizar las pruebas establecidas.

Procedimiento. Esta investigación, se realizó al inicio de la temporada, donde el equipo sub-17, se encontraba en la fase de grupos de la competición; la fecha de realización de las pruebas fue de mutuo acuerdo con el cuerpo técnico del equipo, para no interrumpir en el proceso de entrenamiento y competición. A los deportistas, se les aplicó el test de Bosco, en los saltos Counter Mouvement Jump (CMJ), Repeat, Squat Jump (SJ), Ability Abalakov (ABK) y el test Sprint (RSA), con el fin de analizar las variables de estudio. Los deportistas realizaron los test o fueron evaluados en un solo día, realizando la fase de calentamiento, en la cual, realizaron movimientos articulares, de fuerza y de velocidad, para adaptar a la musculatura del deportista para realizar las pruebas. En la primera prueba, se aplicó el test de potencia del tren inferior y la segunda el test de resistencia a la velocidad. Para el análisis estadístico, se empleó un análisis exploratorio de datos, mediante estadística descriptiva; el procesamiento de la información, se hizo en Microsoft Excel y el software estadístico SPSS
13 en su versión estudiantil, realizando el coeficiente de correlación de Pearson ( $r$ ) datos paramétricos y empleando un alpha mayor o igual a 0,05; además, se procedió a establecer el grado de relación de las mismas en los jugadores de categoría sub-17, de Patriotas Boyacá.

\section{RESULTADOS Y DISCUSIÓN}

En esta sección, se presentan los resultados referidos a este trabajo investigativo y se describen las variables Squat Jump (SJ), Contramovimiento (CMJ) y Abalakov $(A B K)$, asociadas con la potencia del tren inferior $y$, a su vez, guardan relación con el Índice de Fatiga (IF) de los futbolistas de la categoría sub-17, del Club Patriotas Boyacá; los datos correspondientes a estas variables fueron recolectados, a través del test de Bosco.

En el tercero, se explora la potencia, debida al SJ, CMJ y ABK, generada por los futbolistas. En el cuarto, se explora la resistencia a la velocidad, mediante el test Repeat Sprint Ability (RSA), asociada con el IF de los participantes en el estudio. En el quinto, se elabora un análisis correlacional al $5 \%$ de significancia, que permite establecer las correlaciones entre la potencia y la resistencia a la velocidad de los jugadores. Este análisis cuantitativo da cuenta del logro de los objetivos específicos del trabajo investigativo. En cada apartado, se realiza un análisis exploratorio de datos, mediante estadística descriptiva; el procesamiento de la información, se adelantó mediante la hoja de cálculo Excel y el software estadístico SPSS 13, en su versión estudiantil.

Por otro lado, en el análisis descriptivo de las variables involucradas en el estudio, se recurre al uso de distintas medidas estadísticas, como el promedio, la desviación estándar (DS), los porcentajes, el mínimo, el máximo y el coeficiente de variación (CV); el CV, se calcula para variables cuantitativas, dividiendo el valor de la desviación estándar entre el promedio; este resultado, se multiplica por el $100 \%$, con el fin de interpretarlo. El CV corresponde a un indicador de la concentración de los datos de una variable respecto al promedio y permite establecer si los futbolistas son homogéneos (similares) o heterogéneos respecto a la variable que se esté considerando en el análisis cuantitativo de los datos.

De forma general, si el CV es inferior al $8 \%$, se considera que los datos son homogéneos; si el CV está entre el 8 y el $18 \%$, los datos son casi homogéneos; si el CV está 
entre el 18 y el $32 \%$, los datos son casi heterogéneos y si CV es superior al $32 \%$, los datos son heterogéneos (Burbano Pantoja \& Valdivieso Miranda, 2016).

Caracterización respecto al SJ, CMJ y ABK. En esta sección, se efectúa un análisis descriptivo de los jugadores de la categoría sub-17, respecto al Squat Jump (SJ), Contramovimiento (CMJ) y Abalakov (ABK).

Squat Jump. Los jugadores de fútbol de la categoría sub-17 Patriotas Boyacá, quienes participaron en el presente estudio, obtuvieron un salto (Squat jump) mínimo de 31,10 centímetros y un máximo de 45,70, con un promedio de 37,21 $\mathrm{cm}$ y una desviación estándar de 3,72. El coeficiente de variación CV fue del 10,00 \%, lo que indica que los futbolistas resultaron casi homogéneos, en cuanto a este tipo de salto.

Contra-movimiento Jump. Los jugadores obtuvieron un CMJ mínimo de $31,50 \mathrm{~cm}$ y un máximo de 45,30, con un promedio de 37,97, una desviación estándar de 3,66 cm y un CV de 9,65 \%. El CV indica que los futbolistas resultaron casi homogéneos, en cuanto al CMJ.

Abalakov. Los jugadores alcanzaron un ABK mínimo de $33,80 \mathrm{~cm}$ y un máximo de 51,90, con un promedio de 43,27 cm, una desviación estándar de $5,16 \mathrm{~cm}$ y un $\mathrm{CV}$ de $11,93 \%$, indicando que los futbolistas resultaron casi homogéneos, en cuanto al ABK.

En síntesis, el análisis descriptivo reflejó que el grupo de estudio se caracterizó por ser homogéneo, respecto al SJ, CMJ y al $A B K$, por consiguiente, los jugadores de fútbol sub-17 de Patriotas Boyacá son altamente semejantes, en cada uno de estos tipos de saltos.

\section{Caracterización de los futbolistas sub-17} respecto a la potencia debida al SJ, CMJ y

Abalakov. En este apartado, se efectúa un análisis descriptivo sobre las variables referidas a la potencia debida al Squat Jump (SJ), Contramovimiento (CMJ) y Abalakov (ABK), las cuales, están asociadas con la potencia de miembros inferiores de los futbolistas de la categoría sub-17 de Patriotas Boyacá.

Potencia debida al Squat Jump SJ. Los jugadores de fútbol, quienes participaron en este estudio, presentan una potencia debida al Squat Jump, entre 2479,10 y 3630,50 Watts, con un promedio de 2861,94 Watts, una desviación estándar de 314,57 Watts y un CV de
10,99 \%, lo que indica que los futbolistas resultaron casi homogéneos, en lo que se refiere a la potencia debida al SJ.

Potencia debida al CMJ. En esta variable, la potencia debida al CMJ osciló entre 2465,80 y 3585,20 Watts, con un promedio de 2908,27 Watts, una desviación estándar de 317,45 Watts y un CV de 10,91\%, indicando que los futbolistas de la categoría sub-17 son casi homogéneos, en cuanto a la potencia debida al CMJ.

Potencia debida al ABK. Los jugadores de fútbol de la categoría sub-17, quienes participaron en este estudio, presentan una potencia debida al ABK, entre 2540,30 y 3630,50 Watts, con un promedio de 3237,46 Watts, una desviación estándar de 401,99 y un CV de 12,41\%, lo que indica que los futbolistas participantes resultaron casi homogéneos, en cuanto a la potencia debida al ABK.

Además, cinco jugadores que corresponde al 27,78 $\%$ de la muestra lograron una potencia ABK, desde 2540,30 hasta 2975,20 Watts; 11 futbolistas que corresponde al 66,67\%, alcanzaron una potencia ABK, desde 3006.80 hasta 3729.00 Watts y solamente un futbolista, que corresponde al 5,55 \% de la muestra, logró una potencia ABK, de 4156,70. También, se deduce que el $55,56 \%$ de los participantes, se ubicaron por debajo del promedio del grupo de estudio y el restante $44,44 \%$, superó el valor del promedio de la potencia ABK.

En síntesis, el análisis descriptivo evidencia que el grupo de estudio se caracteriza por ser altamente semejante en cuanto a la potencia generada por el SJ, CMJ y ABK. En consecuencia, los jugadores de fútbol participantes en el estudio resultaron similares en cada una de las tres potencias; no obstante, se establece la existencia de un mayor promedio en la potencia $A B K$, seguida de la potencia CMJ y de la potencia SJ, respectivamente.

Índice de fatiga (IF). En la tabla 1, se presentan los resultados referidos al peor tiempo, el mejor tiempo (Sprint) y el IF de los jugadores de fútbol, categoría sub-17 de Patriotas Boyacá, quienes participaron en este estudio. En cuanto al CV, del peor tiempo y el mejor tiempo, los futbolistas resultaron homogéneos en estas variables, mientras para el índice de fatiga (IF) presentó un CV de 62,86\%, lo que indica que los futbolistas son muy heterogéneos para esta característica. 
Tabla 1. Peor y mejor tiempo (Sprint) e Índice de Fatiga (IF) de los jugadores de fútbol sub-17 Patriotas Boyacá.

\begin{tabular}{|l|c|c|c|}
\hline & Peor tiempo & Mejor tiempo & Índice de fatiga \\
\hline Muestra & 18 & 18 & 18 \\
\hline Promedio & 4,98 & 4,38 & 7,16 \\
\hline Desviación estándar & 0,24 & 0,26 & 4,5 \\
\hline Mínimo & 4,63 & 3,90 & 2,27 \\
\hline Máximo & 5,50 & 5,04 & 18,32 \\
\hline
\end{tabular}

\section{Análisis correlacional}

En esta sección, se presenta un análisis correlacional al $5 \%$ de significancia, que permite determinar la existencia de correlaciones entre la potencia y la resistencia a la velocidad de los jugadores, debido a que el IF es un indicador asociado directamente con la resistencia a la velocidad de los futbolistas, por eso, se estudian las correlaciones entre la potencia debida al SJ, CMJ y ABK con el IF y otras características propias de los futbolistas, de la categoría sub-17 del club Patriotas Boyacá.

Las hipótesis para comprobar fueron las siguientes:

- HO: la potencia y el IF no están correlacionadas

- $\quad H 1$ : la potencia y el IF si están correlacionadas de forma significativa

Con la finalidad de analizar las mencionadas correlaciones, se obtuvo la matriz de correlaciones, cuyos componentes sonvalores referidos al coeficiente de correlación de Pearson. En esta matriz, los valores que se ubican por fuera de la diagonal principal son, precisamente, las correlaciones de Pearson. Un valor cercano a 1 es indicativo de la existencia de una relación fuerte y directa entre la potencia y el IF; un valor cercano a 0,5 indica la existencia de una relación moderada y directa; un valor próximo a cero, pero con valores positivos es indicativo de la existencia de una relación débil y directa; un valor cercano a cero, con coeficientes negativos, da cuenta de una relación débil e inversa; un valor próximo a-0.5 es indicativo de una relación moderada e inversa y un valor próximo a-1, da cuenta de la existencia de una relación fuerte e inversa. Las interpretaciones de los valores y las inferencias, se efectuaron con una significancia del $5 \%$.

En la tabla 2, se pueden observar las correlaciones entre el IF y la potencia debida al SJ, CMJ y ABK de los futbolistas, quienes participaron en este estudio.

Con fundamento en la tabla 2 , se deduce que existe

Tabla 2. Matriz de correlaciones entre índice de fatiga y la potencia debida al Repeat Squat Jump-SJ, Counter Mouvement Jump-CMJ y Ability Abalakov-ABK.

\begin{tabular}{|l|l|c|c|c|c|}
\cline { 3 - 5 } \multicolumn{2}{c|}{} & IF & $\begin{array}{c}\text { Potencia } \\
\text { SJ }\end{array}$ & $\begin{array}{c}\text { Potencia } \\
\text { CMJ }\end{array}$ & $\begin{array}{c}\text { Potencia } \\
\text { ABK }\end{array}$ \\
\hline \multirow{4}{*}{ IF } & Correlación de Pearson & 1 & $-0,01$ & 0,01 & 0,14 \\
\cline { 2 - 6 } & 2-colas & & 0,96 & 0,98 & 0,57 \\
\cline { 2 - 6 } & Muestra & 18 & 18 & 18 & 18 \\
\hline \multirow{3}{*}{$\begin{array}{l}\text { Potencia } \\
\text { SJ }\end{array}$} & Correlación de Pearson & $-0,01$ & 1 & $0,96\left(^{* *}\right)$ & $-0,90\left(^{* *}\right)$ \\
\cline { 2 - 6 } & 2-colas & 0,96 & & 0,001 & 0,001 \\
\cline { 2 - 6 } & Muestra & 18 & 18 & 18 & 18 \\
\hline \multirow{3}{*}{$\begin{array}{l}\text { Potencia } \\
\text { CMJ }\end{array}$} & Correlación de Pearson & 0,01 & $0,96\left(^{* *}\right)$ & 1 & $0,88\left(^{* *}\right)$ \\
\cline { 2 - 6 } & 2-colas & 0,98 & 0,00 & & 0,0001 \\
\cline { 2 - 6 } & Muestra & 18 & 18 & 18 & 18 \\
\hline \multirow{2}{*}{$\begin{array}{l}\text { Potencia } \\
\text { ABK }\end{array}$} & Correlación de Pearson & 0,144 & $0,90\left(^{* *}\right)$ & $0,88\left(^{* *}\right)$ & 1 \\
\cline { 2 - 6 } & 2-colas & 0,57 & 0,00 & 0,00 & 18 \\
\cline { 2 - 6 } & Muestra & 18 & 18 & 18 & 18 \\
\hline
\end{tabular}


una correlación no significativa al $5 \%$, entre la potencia debida al SJ y el IF; se obtuvo un p-valor de 0,96 , que resultó superior a $5 \%$. El coeficiente de correlación resultante fue de $r=-0,013, p>0.05,2$ colas, por consiguiente, se puede concluir, que existe una correlación débil e inversa entre la potencia SJ y el IF. Estos resultados evidencian que un incremento en la potencia SJ genera una leve disminución del IF; además, con base en la tabla 2 , se establece que existe una correlación no significativa al $5 \%$, entre la potencia debida al CMJ y el IF, se obtuvo un p-valor de 0,97 , superior al $5 \%$ y el coeficiente de correlación resultante fue de $r=0,007, p>0.05,2$ _colas; por consiguiente, se concluye, que existe una correlación débil y directa entre la potencia CMJ y el IF. Estos resultados evidencian que un incremento en la potencia CMJ produce un leve aumento en el IF.

Se determinó, que existe una correlación no significativa entre la potencia debida al ABK y el IF; se obtuvo un p-valor de 0,56, superior al $5 \%$ y el coeficiente de correlación resultante fue de $r=0,144$, $p>0.05,2$ _colas, por lo tanto, se puede concluir, que existe una correlación débil y directa entre la potencia $A B K$ y el IF. Estos resultados evidencian que hay un incremento en la potencia ABK, lo que implica un leve incremento en el IF. En síntesis, existe una relación leve entre la potencia y el IF, como indicador de la resistencia a la velocidad, es decir, la potencia, sí influye sobre la resistencia a la velocidad, pero no de manera significativa.

El IF obtenido permite interpretar que no es un indicador del rendimiento de los futbolistas sub17 en RSA, porque las diferencias son leves en el rendimiento de la prueba en correspondencia con las demarcaciones; estos hallazgos deben ser tomados en consideración por los entrenadores al momento de planificar y diseñar sus temporadas de competición, con el propósito de optimizar el rendimiento de los jugadores de fútbol, en esta categoría, teniendo presente que la resistencia a la velocidad de los futbolistas de la categoría sub-17, quienes participaron en este estudio, lograron valores poco similares (heterogéneos), en cuanto al IF; sin embargo, se observó una marcada tendencia de los futbolistas a ubicarse en la categoría de excelente $(77,78 \%)$ de IF, en contraste, con un $11,11 \%$, de quienes se ubicaron como buenos o regulares en su IF.

De los resultados presentados en la tabla 2, se pudieron establecer otras correlaciones, como las siguientes. Existe una correlación significativa al $5 \%$ entre la potencia debida al SJ y la potencia debida al CMJ; se obtuvo un p-valor de 0,0001, inferior al $5 \%$ y el coeficiente de correlación resultante fue de $r=$ 0,957, $p<0,05,2$ _colas; por consiguiente, se puede concluir, que existe una correlación fuerte y directa entre estas dos potencias. Estos resultados evidencian que un incremento en la potencia SJ genera un fuerte incremento significativo en la potencia debida al CMJ. Además, con base en la tabla 2 , se establece que existe una correlación significativa al $5 \%$ entre la potencia debida al SJ y la potencia ABK; se obtuvo un $p$-valor de 0,0001, menor que 0,05 y el coeficiente de correlación resultante fue de $r=0,90, p<0,05$, 2_colas; por consiguiente, se concluye que existe una correlación fuerte y directa entre estas dos potencias. Estos resultados evidencian que un incremento en la potencia SJ produce un aumento significativo en la potencia ABK y viceversa.

También, con los resultados de la tabla 2, se determinó que existe una correlación significativa al $5 \%$ entre la potencia debida al CMJ y la potencia ABK; se obtuvo un p-valor de 0,0001, inferior al $5 \%$ y el coeficiente de correlación resultante fue de $r=0,878, p<0,05,2$ colas; por lo tanto, se puede concluir que existe una correlación fuerte y directa entre estas dos potencias. Estos resultados evidencian que un incremento en la potencia CMJ genera un incremento significativo en la potencia debida al ABK y viceversa. En síntesis, existe una relación fuerte y directa entre la potencia debida al SJ, CMJ y ABK, es decir, cada una de las potencias afecta de manera significativa sobre las otras dos.

Adicionalmente, se determinaron las correlaciones entre el mejor tiempo (Sprint) y las potencias debidas al SJ, CMJ y ABK. Los resultados, se pueden observar en la tabla 3. 
Tabla 3. Matriz de correlaciones entre el mejor tiempo y la potencia debida al Squat Jump-SJ, Counter Mouvement Jump-CMJ y Ability Abalakov-ABK.

\begin{tabular}{|l|l|c|c|c|c|}
\cline { 3 - 5 } \multicolumn{2}{c|}{} & $\begin{array}{c}\text { Potencia } \\
\text { SJ }\end{array}$ & $\begin{array}{c}\text { Potencia } \\
\text { CMJ }\end{array}$ & $\begin{array}{c}\text { Potencia } \\
\text { ABK }\end{array}$ & $\begin{array}{c}\text { Mejor } \\
\text { tiempo }\end{array}$ \\
\hline \multirow{2}{*}{$\begin{array}{l}\text { Potencia } \\
\text { SJ }\end{array}$} & Correlación de Pearson & 1 & $0,96\left(^{* *}\right)$ & $0,90\left(^{* *}\right)$ & $-0,09$ \\
\cline { 2 - 6 } & 2-colas & & 0,00 & 0,00 & 0.72 \\
\cline { 2 - 6 } & Muestra & 18 & 18 & 18 & 18 \\
\hline \multirow{2}{*}{$\begin{array}{l}\text { Potencia } \\
\text { CMJ }\end{array}$} & Correlación de Pearson & $0,96\left(^{* *}\right)$ & 1 & $0,88\left(^{* *}\right)$ & $-0,14$ \\
\cline { 2 - 6 } & 2-colas & 0,00 & & 0,00 & 0,57 \\
\cline { 2 - 6 } & Muestra & 18 & 18 & 18 & 18 \\
\hline \multirow{2}{*}{$\begin{array}{l}\text { Potencia } \\
\text { ABK }\end{array}$} & Correlación de Pearson & $0,90\left(^{* *}\right)$ & $0,88\left(^{* *}\right)$ & 1 & $-0,17$ \\
\cline { 2 - 6 } & 2-colas & 0,00 & 0,00 & 18 & 0,513 \\
\cline { 2 - 6 } & Muestra & 18 & 18 & 18 & 18 \\
\hline \multirow{3}{*}{$\begin{array}{l}\text { Mejor } \\
\text { tiempo }\end{array}$} & Correlación de Pearson & $-0,09$ & $-0,14$ & $-0,17$ & 1 \\
\cline { 2 - 6 } & 2-colas & 0,72 & 0,57 & 0,51 & \\
\cline { 2 - 6 } & Muestra & 18 & 18 & 18 & 18 \\
\hline
\end{tabular}

Con base en la tabla 3, se establece que existe una correlación no significativa entre la potencia debida al SJ y el mejor tiempo; por consiguiente, se puede concluir, que existe una correlación débil e inversa entre la potencia SJ y el mejor tiempo; estos resultados evidencian que un mejor tiempo implica una leve disminución en la potencia SJ. Además, con fundamento en la tabla 3, se establece que existe una correlación no significativa entre el mejor tiempo y potencia debida al CMJ, por lo que se deduce, que existe una correlación débil e inversa entre el mejor tiempo y la potencia CMJ; estos resultados evidencian que un mejor tiempo produce un leve decremento en la potencia CMJ.

También, con los resultados de la tabla 3, se determinó que existe una correlación no significativa entre el mejor tiempo y la potencia debida al ABK, por lo tanto, se puede concluir, que existe una correlación débil e inversa entre el mejor tiempo y la potencia ABK; estos resultados evidencian que un mejor tiempo implica una leve disminución en la potencia ABK. En síntesis, existe una relación leve entre la potencia y el mejor tiempo asociado con la velocidad de desplazamiento, es decir, la velocidad de desplazamiento sí influye sobre la potencia, pero no de forma significativa.

Los resultados que se pueden evidenciar son respecto a las variables que fueron potencia y resistencia, midiéndose a través del test Bosco, con relación a la potencia, en cuanto a la ejecución de los saltos, siendo $A B K$, SJ y CMJ, razón, por la que, a partir de esto, se evidencia que el test Bosco contribuye a la comprensión de la potencia que pueden tener los jugadores de fútbol y esto es comparado con lo que afirman Salinero et al. (2013), dado que valoran la condición física y el nivel técnico que tienen los futbolistas, entre los 10 y los 17 años.

Relacionándose con la investigación que hicieron Méndez Galvis et al. (2007), en lo correspondiente con la fuerza en el desarrollo que tiene en la potencia en los jugadores que, a partir de allí, se puede relacionar la efectividad y el uso de estas pruebas para dar cuenta de las dos variables y, por tanto, fortalecer las investigaciones que se hacen alrededor del fútbol.

Ahora bien, los resultados del test de Bosco en (SJ), tuvo un promedio de $37,21 \mathrm{~cm}$, con un CV del $10 \%$, un ABK medio de 43,27 cm, con un CV del 11,93\% y, respecto al CMJ, tuvo una media de $37,97 \mathrm{~cm}$, con un CV de 9,65\%. En cuanto al test RSA, se indica el índice de fatiga de los deportistas, con una media de 7,16, con una desviación estándar de 4,50 \% y un CV de 62,86\%, lo que indica que los deportistas son heterogéneos, en lo que tiene que ver con el índice de fatiga $y$, ello muestra, que no todos los futbolistas tienen los mismos valores, de acuerdo con la clasificación general de los test.

De acuerdo con los resultados obtenidos, se evidencia que no hay una correlación entre los indicadores del 
test RSA y de los altos ABK y SJ y, a su vez, el contramovimiento $\mathrm{CMJ}$ tiene correlación con los índices de fatiga, potencia y sprint, comparándose con los estudios de Sánchez-Sánchez et al. (2005), quienes afirman que los jugadores de fútbol de sala no tuvieron resultados similares, porque no hay una correlación significativa entre el contra - movimiento CMJ y el test RSA. Cabe mencionar que, de acuerdo con los resultados del RSA, se muestra una leve pérdida en el rendimiento de los jugadores en el tiempo, debido a que, a medida que pasan las repeticiones en sprint, éste disminuye, siendo parecido a la investigación de Yanci Irigoyen et al. (2017), en donde no encontraron diferencias significativas entre los test RSA y RECODA.

Ahora bien, sí hay una correlación entre SJ y el mejor tiempo, de acuerdo con lo que se muestra en el test RSA, por lo que, comparado con los estudios presentados anteriormente, no se obtienen los mismos resultados, pero sí tiene coincidencia en que uno de los saltos se correlaciona y ello puede ser debido a las características del deporte y, a su vez, del deportista.

Dal Pupo et al. (2014) realizaron un estudio a jugadores de sala en Brasil, en las categorías sub15 y sub-17, en el cual, comparó la potencia y la resistencia de los jugadores, teniendo como objetivo principal correlacionar status variable y, con ello, obtuvo de las muestras que sí hubo una correlación significativa entre los saltos y los sprints, teniendo la mayor puntuación el CMJ y el tiempo medio en línea recta. Estos resultados son similares a los propios de esta investigación, con concordancia, a su vez, con las edades a las cuales se investigó; por tanto, se puede decir que, debido a los resultados obtenidos y comparándolo con el estudio de Dal Pupo et al. (2014), estos futbolistas tienen una buena condición física, de acuerdo con su edad.

Respecto al porcentaje de fibras rápidas y según la utilización de éstos, se evidencia que la fuerza explosiva de los miembros inferiores, que tienen que ver con el contra-movimiento, hay aspectos que se reflejan en las investigaciones presentadas anteriormente. Por otra parte, según el estudio de Urzua (2009), en cuanto a las características fisiológicas desde el desempeño aeróbico, anaeróbico, la fuerza y la potencia muscular, se evidencian datos significativos, de acuerdo con el test de Bosco, que fue aplicado a jugadores profesionales y universitarios, resultando con ello, una poca significancia entre las dos variables y sirviendo esta investigación, como referente para la presente.

En cuanto al índice de fatiga, se encontró un leve aumento y es posible compararlo con resultados de investigaciones, como la de Rodríguez-Fernández et al. (2014), en la cual, en ambas investigaciones, los resultados fueron similares con los obtenidos en esta investigación y de acuerdo con las edades. Igualmente, según los resultados obtenidos en CMJ, hay que mencionar que éstos fueron por debajo de los encontrados en otras investigaciones, como las de Silva et al. (2012), pero muy similares a los obtenidos por García Manso et al. (1999).

En lo que tiene que ver con SJ, se puede decir que los datos fueron superiores, si se compara con los de García Manso et al. (1999), dado que se puede deber a las diferencias de edades y al nivel de entrenamiento que tenía cada equipo, así como el tiempo que llevaron a cabo para la realización de la investigación. Así, las cosas, se puede decir, que los resultados obtenidos en comparación con otras investigaciones son similares, pero, a su vez, tienen diferencias significativas, en cuanto a los resultados y esto se puede deber a diversos factores, como la edad, el tiempo de entrenamiento, los entrenadores y la heterogeneidad del equipo.

Por tanto, es posible decir, que se acepta la hipótesis nula y se rechaza la hipótesis alternativa, debido a que no existe relación entre potencia y resistencia a la velocidad de los jugadores de fútbol de la categoría sub-17, del equipo Patriotas Boyacá.

\section{CONCLUSIONES}

Con fundamento en los hallazgos asociados al análisis descriptivo, se logró establecer que el grupo de estudio, conformado por los futbolistas de la categoría sub-17 de Patriotas Boyacá, se ha caracterizado por ser homogéneo, en lo referente a las variables estatura e IMC, pero casi homogéneo, en cuanto al peso; por lo tanto, los futbolistas de esta categoría resultaron altamente semejantes en las mencionadas características. Estos hallazgos proporcionaron elementos contextuales semejantes para continuar hacia su caracterización, en cuanto a su SJ, CMJ, ABK y sus potencias respectivas; asimismo, permitió el análisis de su IF. 
El análisis descriptivo permitió evidenciar que el grupo de estudio resultó casi homogéneo en lo referente al SJ, CMJ y al ABK, por lo tanto, los jugadores de fútbol sub-17 de Patriotas Boyacá son altamente semejantes, en cada uno de estos tipos de saltos; no obstante, se determinó que había un mayor promedio en el ABK, seguido del CMJ y del SJ, respectivamente.

Del análisis exploratorio, se estableció que el grupo de estudio se ha caracterizado por ser bastante similar, en cuanto a la potencia generada por el SJ, CMJ y ABK, por consiguiente, los jugadores de fútbol participantes en el estudio resultaron semejantes en cada una de las tres potencias; sin embargo, se determina la existencia de un mayor promedio en la potencia $A B K$, seguida de la potencia CMJ y de la potencia SJ, respectivamente.

Los resultados permiten concluir, que los participantes en el grupo de estudio son muy heterogéneos, con un índice de fatiga promedio de 7,16, es decir, el grupo es altamente heterogéneo, en cuanto al IF. Este índice, se pudo afectar, porque el peor tiempo de sprint varió entre 4,63 y 5,50 segundos; en cambio, el mejor tiempo varió entre los 3,90 y 5,04 segundos, tiempos que se podrían considerar grandes, para el recorrido ejecutado.

Finalmente, del análisis correlacional, se concluye que en los futbolistas del grupo de estudio existe una relación leve entre la potencia y el IF, como indicador de la resistencia a la velocidad; lo anterior indica, que la potencia sí influye sobre la resistencia a la velocidad, pero no de manera significativa. Adicionalmente, existe una relación fuerte y directa entre la potencia debida al SJ, CMJ y ABK, lo cual, es indicativo para que cada una de las potencias afecte, de manera significativa, sobre las otras dos; un incremento de una de ellas, genera un aumento significativo en las demás.

Conflicto de intereses: El manuscrito fue preparado y revisado con la participación de todos los autores, quienes declaramos que no existe ningún conflicto de intereses que ponga en riesgo la validez de los resultados presentados. Además, es importante mencionar que la presente investigación, se encuentra anexa al macroproyecto "Modelos y estrategias determinantes en el entrenamiento deportivo" de la Maestría en Pedagogía de la Cultura Física, de la Universidad Pedagógica y Tecnológica de Colombia.

\section{REFERENCIAS}

1. ÁlvareZ medinA, J.; GIMÉNEZ SALILLAS, L.; CORONAVIRÓN, P.; MANONELLESMARQUETA, P. 2002. Necesidades cardiovasculares y metabólicas del fútbol sala: análisis de la competición. Apunts Educación Física y Deportes. 67:45-51.

2. BURBANO PANTOJA, V.M.; VALDIVIESO MIRANDA, M.A. 2016. Inferencia estadística básica, apoyo al estudio independiente. Editorial UPTC. Tunja, Colombia. 222p.

3. DAL PUPO, J.; DETANICO, D.; DOS SANTOS, S.G. 2014. The fatigue effect of a simulated futsal match protocol on isokinetic knee torque production. Sport Biomechanics. 13(4):332340.https://doi.org/10.1080/14763141.2014 .981202

4. GARCÍA MANSO, J.M.; NAVARRO, M.; RUÍZ CABALLERO, J.A. 1999. Pruebas para la valoración de la capacidad motriz en el deporte. Evaluación de la condición física. Editorial Gymnos. Madrid.

5. LE GALL, F.; BEILLOT, J.; ROCHCONGAR, P. 2002. Évolution de la puissance maximale anaérobie au cours de la croissance chez le footballeur. Sience \& Sports. 17:177-188.

6. LÓPEZ, J. 2017. Manual de la UEFA para entrenadores de Futsal. UEFA. 103p. Disponible desde Internet en: https:// editorial.uefa.com/resources/025dOf842ced7bbc-db8606c7d9fa-1000/manual_ de_entrenamiento_de_futbol_sala_de_ la_uefa.pdf (con acceso el 15 de marzo del 2021).

7. MÉNDEZ GALVIS, E.A.; MARQUÉS ARABIA, J.J.; CASTRO CASTRO, C.A. 2007. El trabajo de fuerza en el desarrollo de la potencia en futbolistas de las divisiones menores de un equipo profesional de fútbol.iatreia. 20(2):127-143.

\section{RODRÍGUEZ-FERNÁNDEZ, A.; SÁNCHEZ-SÁNCHEZ,} J.; VILLA-VICENTE, J.G. 2014. Evolución del rendimiento en la habilidad de repetir sprints 
(RSA) según el momento de la temporada y en función de la demarcación en jóvenes futbolistas. Revista de Preparación Física en el Fútbol.

9. SALINERO, J.J.; GONZÁLEZ-MILLÁN, C.; RUÍZVICENTE, D.; ABIÁN VICÉN, J.; GARCÍAAPARICIO, A.; RODRÍGUEZ-CABREO, M.; CRUZ, A. 2013. Valoración de la condición física y técnica en futbolistas jóvenes. Revista internacional de medicina y ciencias de la actividad física y el deporte. 13(50):401-418.

10. SÁNCHEZ-SÁNCHEZ, J.; BLÁZQUEZ HERNÁNDEZ, F.; GONZALO MARTÍN, A.; YAGÜE CABEZÓN, J.M. 2005. La resistencia a la velocidad como factor condicionante del rendimiento del futbolista. Apuntes Educación Física y Deportes. 81:47-60.

11. SILVA, J.F.; DETANICO, D.; FLORIANO, L.T.; DITTRICH, N.; NASCIMENTO, P.C.; SANTOS, S.G.; GUGLIELMO, L.G.A. 2012. Níveis de potência muscular em atletas de futebol e futsal em diferentes categorías e posições. Motricidade. 8(1):14-22. https://doi.org/10.6063/motricidade.233
12. TERRADOS, N.; CALLEJA-GONZÁLEZ, J.; SCHELLING, X. 2011. Bases fisiológicas comunes para deportes de equipo. Revista Andaluza de Medicina del Deporte. 4(2):8488.

13. URZUA, R.; VON OETINGER, A.; CANCINO, J. 2009. Potencia aeróbica máxima, fuerza explosiva del miembro inferior y peak de torque isocinético en futbolistas chilenos profesionales y universitarios. Kronos Rendimiento en el Deporte. 8(14):49-52.

14. YANCI IRIGOYEN, J.; AZCARATE, U.; LOS ARCOS, A. 2017. Análisis de la capacidad de realizar esprines repetidos con y sin cambio de dirección en futbolistas profesionales. Sport TK: Revista Euroamericana de Ciencias del Deporte. 6(1):51-56. 